\title{
Tracking moving targets in wireless sensor networks using extended diffusion strategies of distributed Kalman filter
}

\begin{abstract}
Using wireless sensor networks to track the position of a moving object in a 3-D spatial model requires precise information of location and speed of the object, which in turn demands for accuracy in state estimation of distributed Kalman filter. In view of reducing the impacts of noise in the dynamic linear system and achieve optimized state estimate, the current study proposes extended strategies of Kalman filter diffusion based on distributed Kalman filter. Through the proposed strategies, each node communicates merely with its neighbor nodes. The data aggregation is done in a set of neighborhood using instructions of recursive Kalman filter iterations with specific weights. The proposed algorithms provide precise state estimates in a moment as global state estimates using various updates at each step. As a simulation study, we applied the algorithms in a network to track the position and speed of a projectile and compared the results with real world circumstances, using the concept of transient mean square deviations of network as a cost function. The results report improvements over the conventional methods in terms of mean square errors.
\end{abstract}

Keyword: Adaptive sensor networks; Diffusion strategies; Distributed estimation; Kalman filter; Target tracking 